Supporting Information for

\title{
Hydrophobic Carbon Dots from Aliphatic Compounds with One Terminal Functional Group
}

Keyang Yin,,${ }^{\dagger} \|$ Dandan Lu, ${ }^{\dagger}$ Liping Wang, ${ }^{*, \S}$ Quanxin Zhang, ${ }^{\dagger}$ Junying Hao, ${ }^{*, \dagger}$ Gaozhan Li, ${ }^{\dagger}$

$$
\text { Hongguang } \mathrm{Li}^{*},
$$

$\dagger$ State Key Laboratory of Solid Lubrication, Lanzhou Institute of Chemical Physics, Chinese Academy of Sciences, Lanzhou 730000, China

$\$$ Key Laboratory of Colloid and Interface Chemistry, Shandong University, Ministry of Education, Jinan 250100, China

$\S$ College of Materials Science and Engineering, Liaocheng University, Liaocheng 252059, China

" University of Chinese Academy of Sciences, Beijing 100049, China

Corresponding authors:

+86-635-8230919. E-mail: wangliping5@ 163.com (Liping Wang)

+86-931-4968236. E-mail: jyhao@licp.cas.cn (Junying Hao)

+86-531-88363597. E-mail: hgli@ sdu.edu.cn (Hongguang Li) 


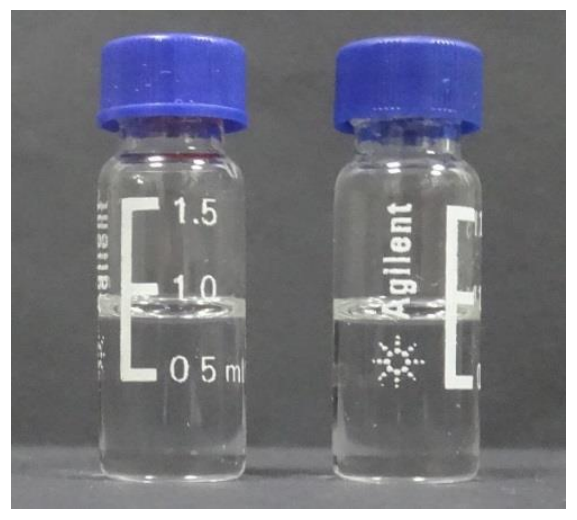

Figure S1. Photos of CB before (left) and after (right) refluxing for $48 \mathrm{~h}$.
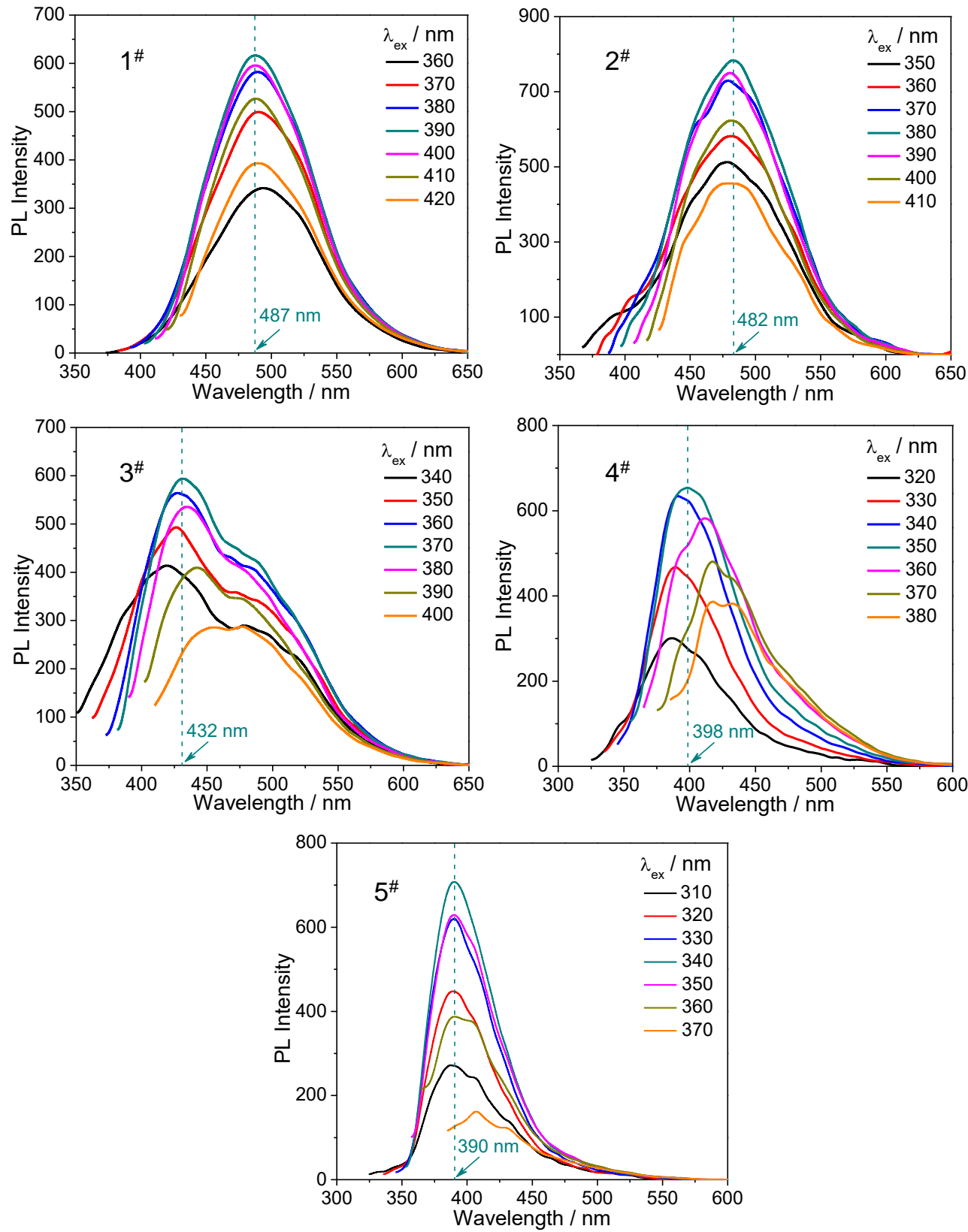

Figure S2. Emission of the C dots produced by 3,4,5-trioctadecyloxybenzaldehyde followed by separation with GPC. Also shown are the wavelengths at which maximum emissions were recorded. The dashed lines are guides for the eyes. 

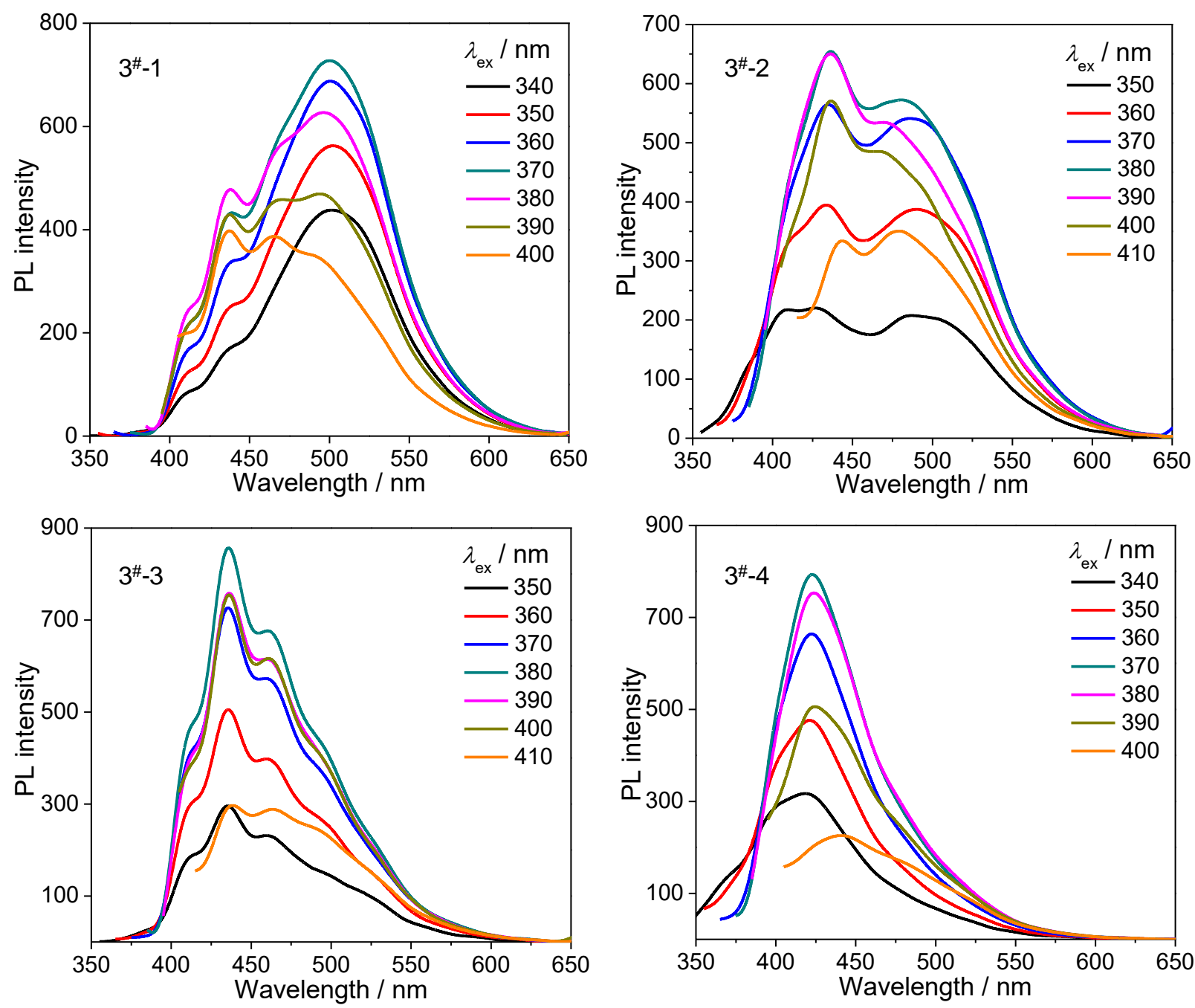

Figure S3. Emission of the C dots obtained by the second round GPC on $3^{\#}$ shown in Figure S2. Totally four fractions were collected, which were arranged following the order of $R_{\mathrm{t}}$.
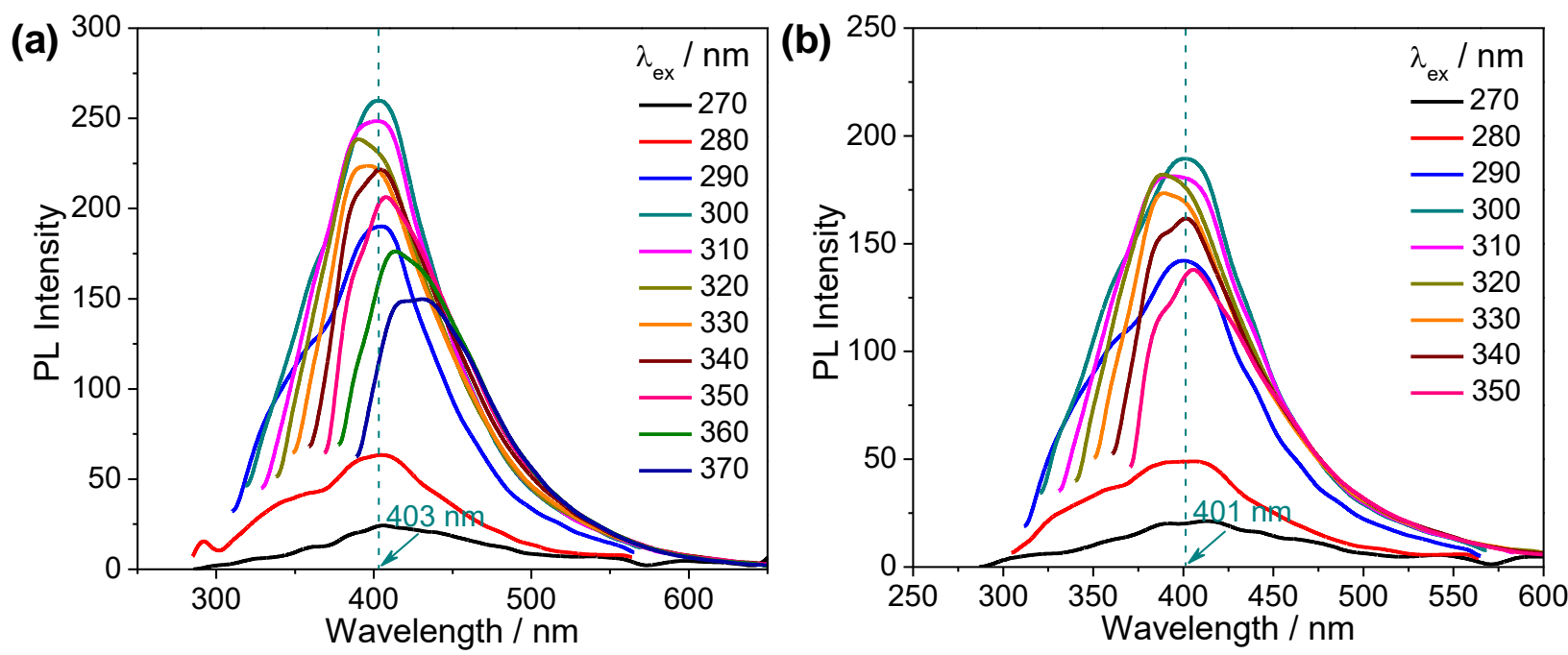

Figure S4. Emission of the as-prepared $\mathrm{C}$ dots from $\mathrm{C}_{12}-\mathrm{OH}$ (a) and $\mathrm{C}_{11}-\mathrm{COOH}$ (b), respectively, at different excitation wavelengths as indicated. Also shown are the wavelengths at which maximum emission were recorded. The dashed lines are guides for the eyes. 

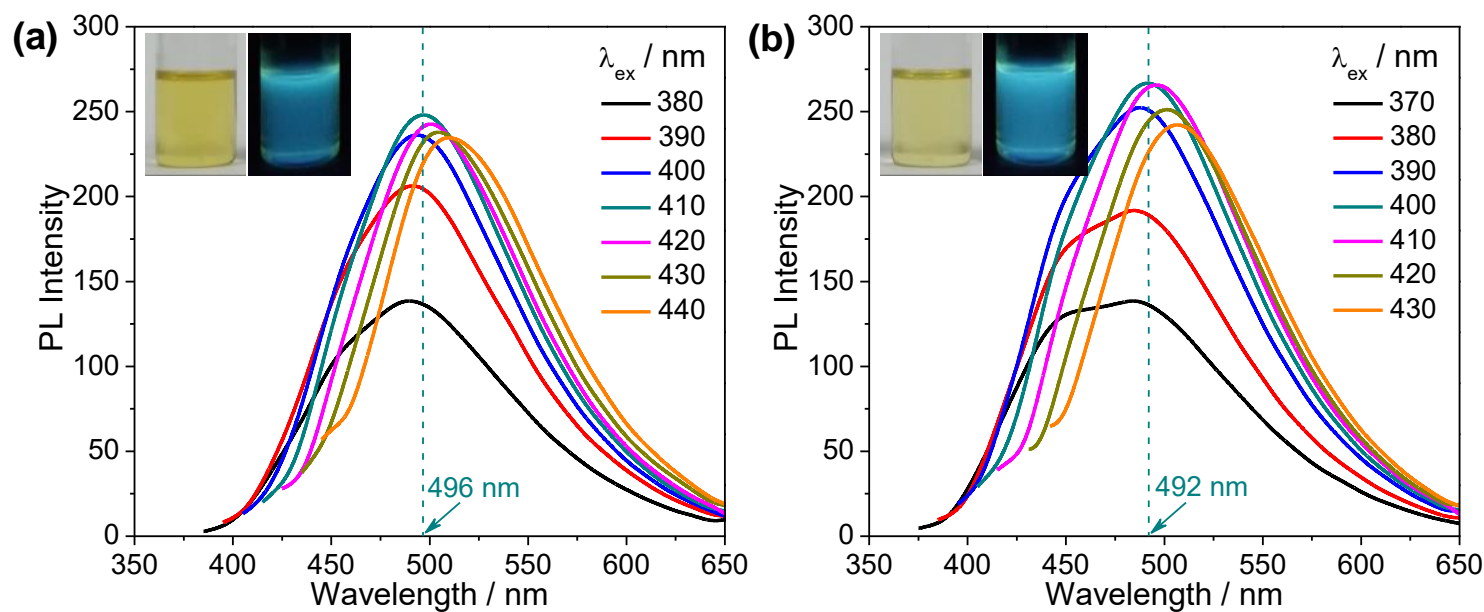

Figure S5. Emission at different excitation wavelengths of the $\mathrm{C}$ dots prepared by refluxing 1.0 mol $\cdot \mathrm{L}^{-1} \mathrm{C}_{12}-\mathrm{NH}_{2}$ (Sigma, $\geq 99 \%$ ) in CB from Sigma (a, analytical grade) and Aladdin (b, analytical grade) for $48 \mathrm{~h}$. The dashed lines are guides for the eyes. Insets in each figure are photos of the as-prepared C dots under room light (left) and $365 \mathrm{~nm}$ UV irradiation (right).
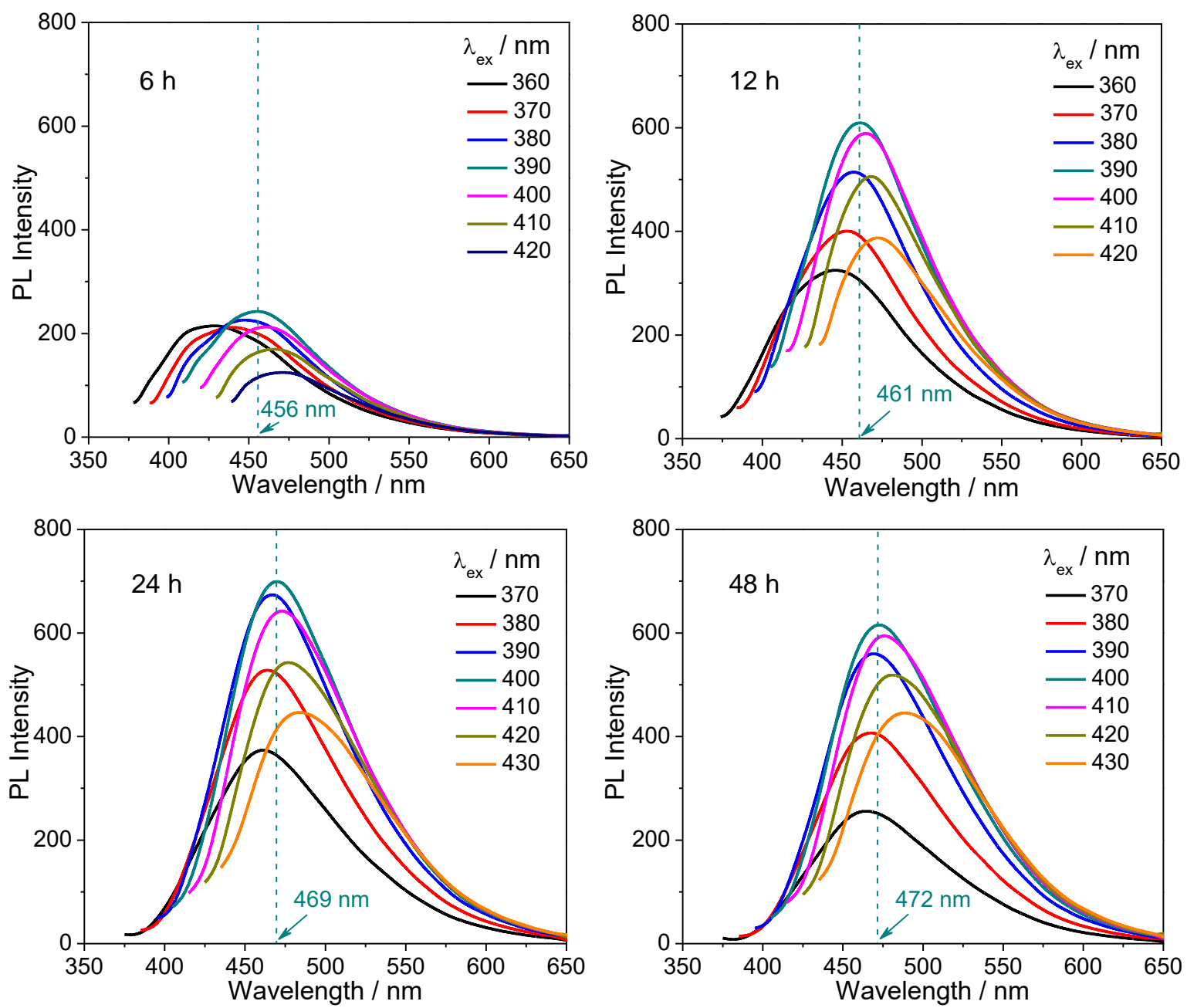

Figure S6. Emission of the $\mathrm{C}$ dots in $\mathrm{CB}$ prepared by refluxing $1.0 \mathrm{~mol} \cdot \mathrm{L}^{-1} \mathrm{C}_{12}-\mathrm{NH}_{2}$ for different time as indicated. The dashed lines are guides for the eyes. 

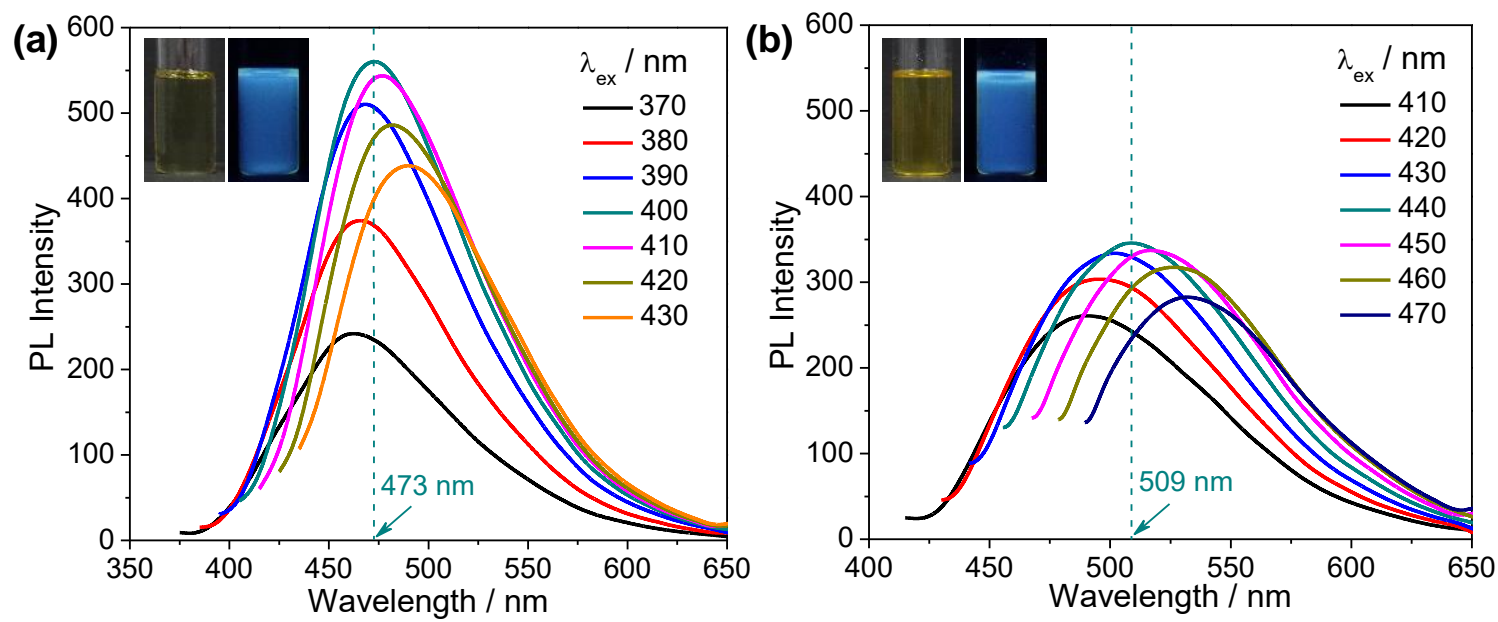

Figure S7. Emission of the as-prepared $\mathrm{C}$ dots from $\mathrm{C}_{8}-\mathrm{NH}_{2}$ (a) and $\mathrm{C}_{16}-\mathrm{NH}_{2}$ (b), respectively, at different excitation wavelengths as indicated. Also shown are the wavelengths at which maximum emission were recorded. The dashed lines are guides for the eyes. Insets in each figure are photos of the as-prepared $\mathrm{C}$ dots under room light (left) and $365 \mathrm{~nm}$ UV irradiation (right).
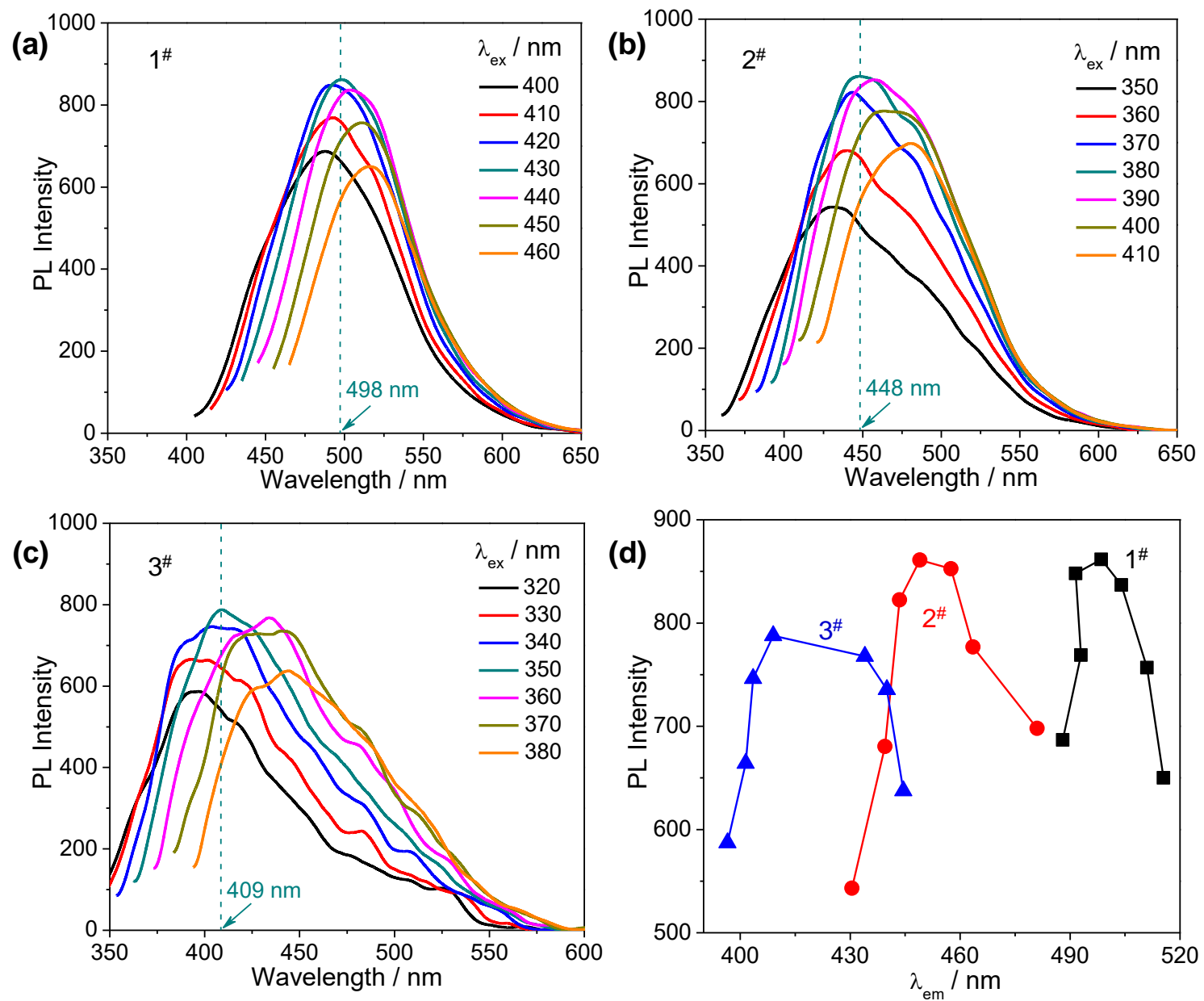

Figure S8. (a-c) Emission of the samples separated by GPC on the as-prepared C dots by refluxing $1.0 \mathrm{~mol} \cdot \mathrm{L}^{-1} \mathrm{C}_{12}-\mathrm{NH}_{2}$ in $\mathrm{CB}$ for $24 \mathrm{~h}$. The samples $1^{\#-3^{\#}}$ correspond to those shown in Fig. 3a. The dashed lines are guides for the eyes. (d) PL intensity and corresponding $\lambda_{\text {em }}$ for samples $1^{\#-3^{\#}}$. Emissions were recorded with a $\lambda_{\text {ex }}$ range of $400-460 \mathrm{~nm}$ for sample $1^{\#}, 350-410 \mathrm{~nm}$ for sample $2^{\#}$, and 320-380 $\mathrm{nm}$ for sample $3^{\#}$ with a step of $10 \mathrm{~nm}$. 


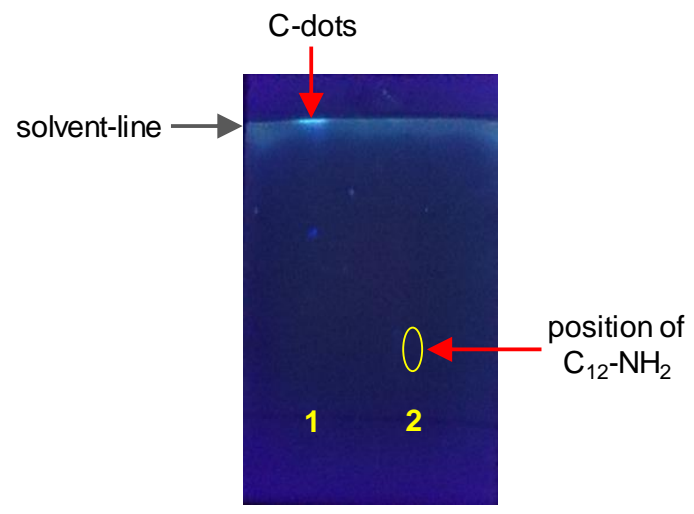

Figure S9. Photos of the silica gel plate eluted with as-prepared $\mathrm{C}$ dots (1) and $\mathrm{C}_{12}-\mathrm{NH}_{2}(\mathbf{2})$ in ethanol. Taken under $365 \mathrm{~nm}$ UV irradiation.
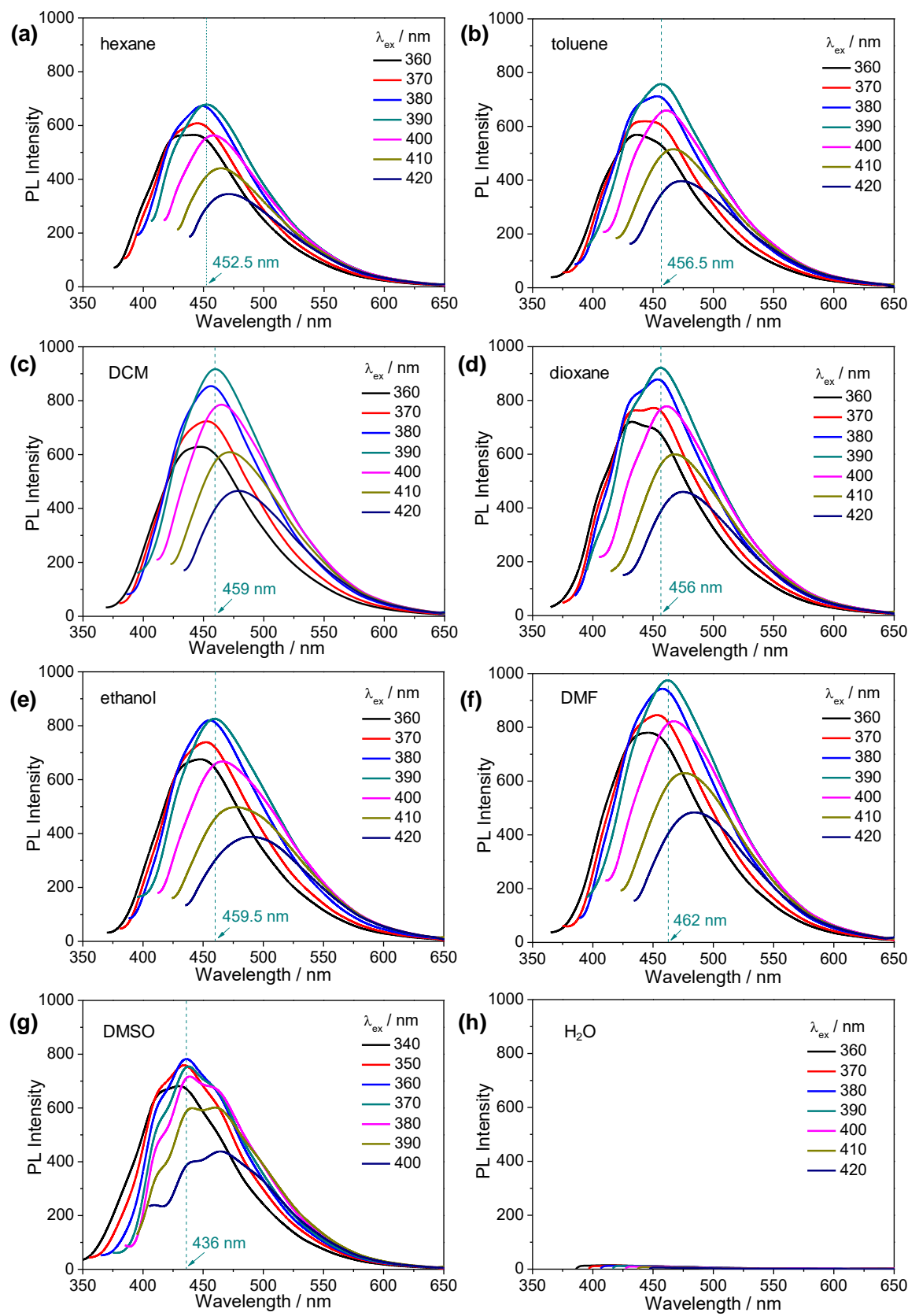

Figure S10. Emission at varying $\lambda_{\text {ex }}$ of $\mathrm{C}$ dots in different solvents. The raw $\mathrm{C}$ dots were prepared by refluxing $1.0 \mathrm{~mol} \cdot \mathrm{L}^{-1} \mathrm{C}_{12}-\mathrm{NH}_{2}$ in $\mathrm{CB}$ for $48 \mathrm{~h}$ followed by purification with silica gel chromatography using ethanol as an eluent. The concentration of $\mathrm{C}$ dots is $3.0 \mathrm{mg} \cdot \mathrm{mL}^{-1}$ in hexane, toluene, DCM, dioxane, ethanol and DMF, or saturated in DMSO and $\mathrm{H}_{2} \mathrm{O}$. 


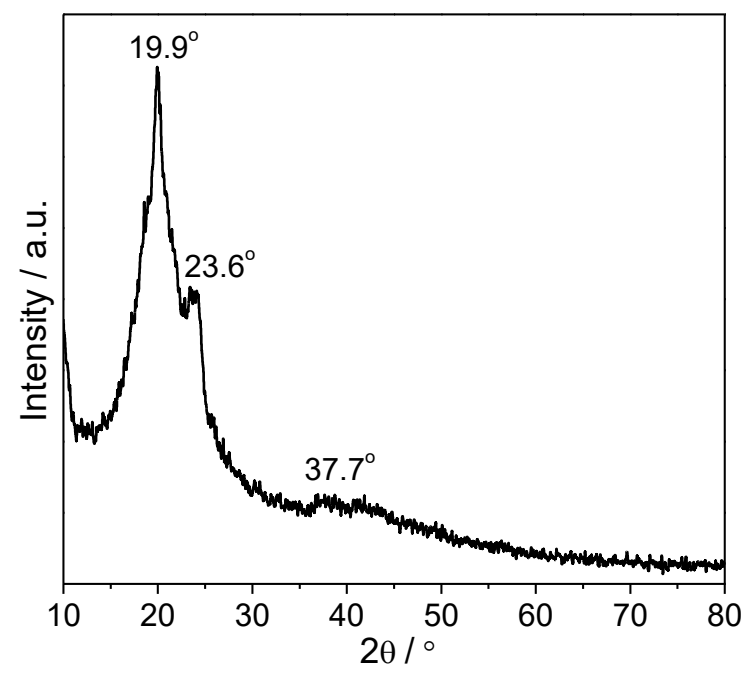

Figure S11. XRD pattern of the C dots.

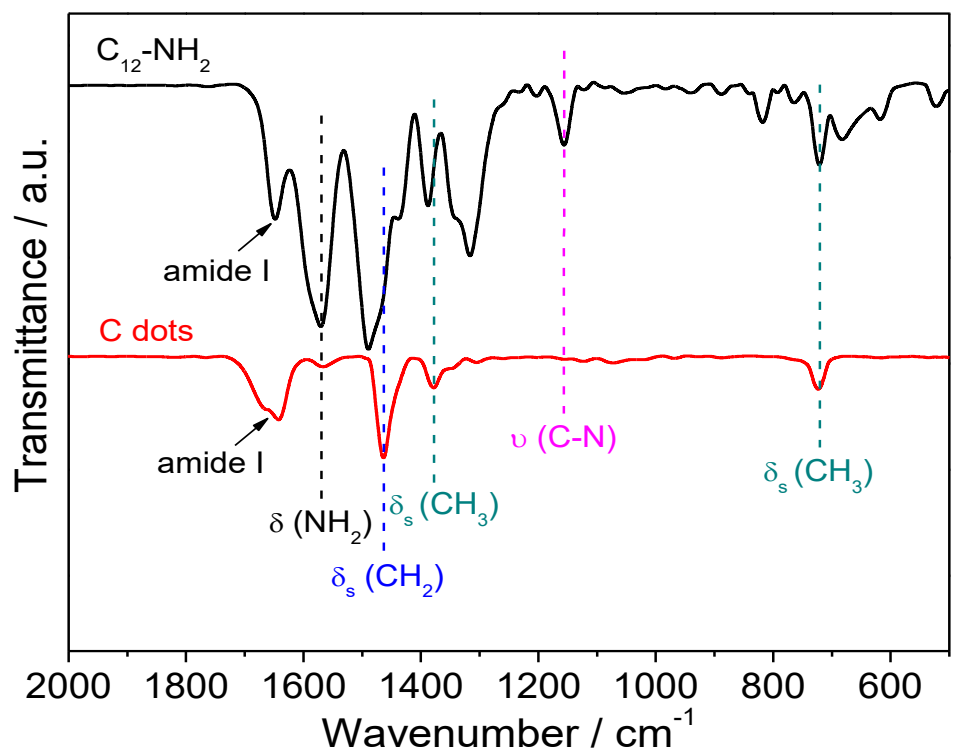

Figure S12. FTIR spectra of $\mathrm{C}_{12}-\mathrm{NH}_{2}$ and $\mathrm{C}$ dots in the range of $2000-500 \mathrm{~cm}^{-1}$. Special peaks have been marked. The dotted lines are guides for the eyes.

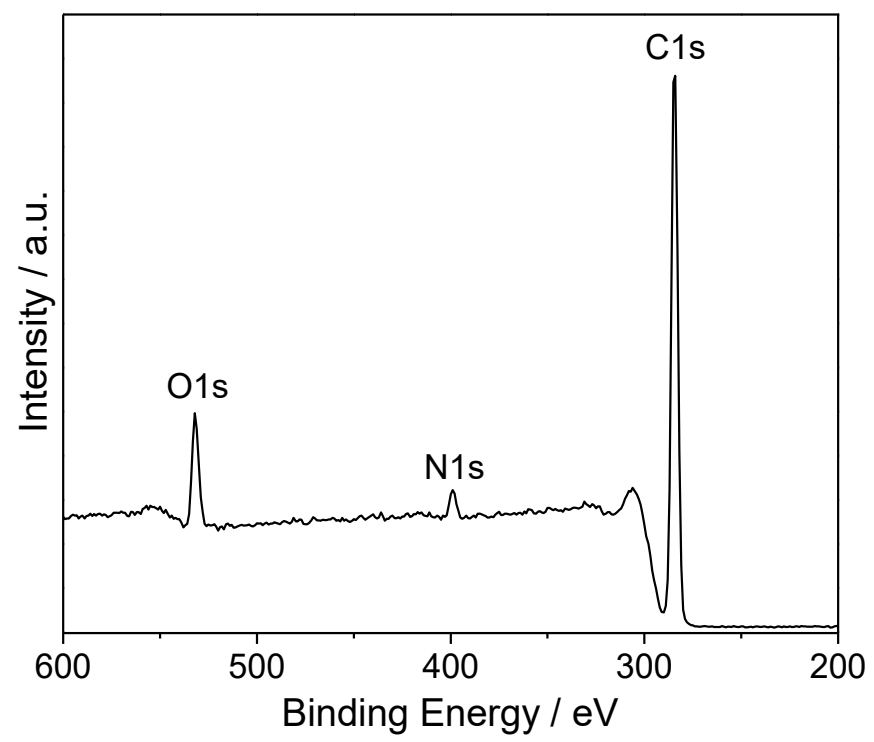

Figure S13. XPS survey of the C dots. 
Table S1. $\lambda_{\mathrm{ex}}$ at which the maximum emission occurs, $\lambda_{\mathrm{em}}$ and PL intensity at $\lambda_{\mathrm{em}}$ of the purified C dots $\left(3.0 \mathrm{mg} \cdot \mathrm{mL}^{-1}\right)$ in different solvents. The raw $\mathrm{C}$ dots were prepared by refluxing $1.0 \mathrm{~mol} \cdot \mathrm{L}^{-1}$ $\mathrm{C}_{12}-\mathrm{NH}_{2}$ in $\mathrm{CB}$ for $48 \mathrm{~h}$ followed by purification with silica gel chromatography using ethanol as an eluent.

\begin{tabular}{cccccccc}
\hline & hexane & toluene & DCM & dioxane & ethanol & DMF & DMSO \\
\hline$\lambda_{\mathrm{ex}} / \mathrm{nm}$ & 390 & 390 & 390 & 390 & 390 & 390 & 360 \\
$\lambda_{\mathrm{em}} / \mathrm{nm}$ & 452.5 & 456.5 & 459 & 456 & 459.5 & 462 & 436 \\
$\begin{array}{l}\text { Maximum PL } \\
\text { intensity / a.u. }\end{array}$ & 678.0 & 757.6 & 916.7 & 921.2 & 825.6 & 974.8 & 782.2 \\
\hline
\end{tabular}

